\title{
Surface Flow Measurements From Drones
}

\author{
Flavia Tauro ${ }^{\mathrm{a}}$, Maurizio Porfiri ${ }^{\mathrm{b}}$, Salvatore Grimaldi ${ }^{\mathrm{a}, \mathrm{b}, 1, *}$ \\ ${ }^{a}$ Dipartimento per l'Innovazione nei sistemi Biologici, Agroalimentari e Forestali, \\ University of Tuscia, Viterbo, Italy. \\ ${ }^{b}$ Department of Mechanical and Aerospace Engineering, New York University Tandon \\ School of Engineering, Brooklyn, NY 11201, USA.
}

\section{Abstract}

Keywords: Flow measurement, Surface Hydrology, Large Scale Particle Image Velocimetry, Unmanned Aerial Vehicle

\footnotetext{
*Corresponding author

Email address: salvatore.grimaldi@unitus.it (Salvatore Grimaldi)

${ }^{1}$ Ph.: +39 0761357326 ; Fax: +390761 357356
}

Preprint submitted to Elsevier

April 13, 2016 


\section{Introduction}

Surface waters influence the way the landscape evolves, ecosystems develop, environmental risks arise, and epidemics propagate [1, 2, 3, 4]. Due to the complexity of surface water processes, a unique observational method for identifying and quantitatively monitoring flows is yet to be found [5]. Traditionally, surface flows are invasively monitored using current meters [6], tracing systems [7], and acoustic doppler instrumentation [8]. Water control structures, such as weirs and spillways, can also be used to estimate flow discharge [9].

Even if the efficacy of such methods has been demonstrated in the technical literature [10], their use is limited to easy-to-access environments. In addition, the measurement accuracy can be affected by the severity of natural rainfall events and by systematic errors associated with sensors interfering with the flow. To address some of these challenges, considerable efforts have been devoted toward the development and refinement of remote methods, including hand-held radars [11], microwave sensors [12], and satellites [13]. These approaches have highly benefitted the realm of hydrological observations; however, most of them are only applicable to large scale channel flows, may be expensive, and may not be suitable to frequently monitor water bodies.

To mitigate such issues, several methods based on the remote acquisition and analysis of flow images have been proposed and implemented in the last two decades [14]. Specifically, in [15], images of large scale riverine ecosystems are analyzed through high-speed cross-correlation to obtain surface flow velocity maps. These surface flow velocity maps can be complemented with 
information on the bathymetry to allow for flow discharge estimations [16]. Such optical observational methods are challenged by practical difficulties, such as the need for collecting ground reference points [17], and, therefore, flow data are often not available at ungauged sites, such as extra-urban areas, large-scale lakes and glaciers, coasts, and river estuaries. Despite practical limitations, optical methods are inherently suited to enable continuous and remote observations over diverse water bodies, spanning from rills to large scale rivers. Notably, the potential of such image-based methods has been assessed on a mountainous stream [18], a semi-natural hillslope [19], and a large scale river [17].

Here, we propose a novel, fully remote approach for surface flow observations that overcomes practical difficulties related to the implementation of optical methods in difficult-to-access environments. Specifically, we put forward the integration of optical observational techniques and drones for non-invasive quantitative flow measurement. With more than six billion dollars a year spent in research and development around the world [20], drones have transformed our capacity to remotely sense and access the environment $[21,22,23,24]$. For instance, in the last decade, drones have empowered multiple fields of science by enabling data collection in hostile environments, such as volcanoes [25] and ice sheets [26], and by providing footage over extended areas, such as forests [22] and natural reserves [27]. Just as aerial and satellite sensing have transformed scientific observations, allowing the resolution of large-scale physical processes [28, 29], the pervasive use of drones is set to revolutionise geophysical sciences through the rapid and refined measurement of small to medium scale phenomena. 
In the realm of hydrology, drones have the potential to enable remote and distributed flow measurements in difficult-to-access water environments during adverse hydro-meteorological events. In our vision, a drone is deployed in natural environments and is remotely piloted (or, alternatively, guided through GPS waypoint trajectory) above the water body of interest upon request/need of the operator. An onboard camera oriented with its axis perpendicular to the water surface captures videos of the water surface, whereby a ground user identifies a region of interest through a wireless monitor in real time. Once the ground user selects an area of interest, the drone is set to the hovering mode, and the platform stationkeeps above the region while taking high-resolution videos of the water surface. Pictures extracted from the drone footage can be processed off-line through several optical-based algorithms to extract the surface velocity field.

To demonstrate the potential of our approach, here, we present the design and preliminary assessment of a novel aerial sensing platform. The platform features a low-cost recreational drone equipped with a miniature camera and a system of lasers to remotely assign metric dimensions to images (remote photometric calibration in the rest of the note) without the acquisition of ground reference points. To assess the feasibility of the hovering capability of the drone for reliable flow velocimetry, we execute preliminary experiments on an ad hoc developed outdoor controlled facility at University of Tuscia. Finally, we perform surface flow observations in a small scale stream (less than $1 \mathrm{~m}$ wide and a few centimeters deep) in the Italian Alps. Interestingly, few noninvasive techniques are currently available to monitor small scale surface flows in natural environments. We remotely capture and calibrate videos 
of the stream from the drone and apply the Large Scale Particle Image Velocimetry (LSPIV) high-speed cross-correlation algorithm [16] that extracts usable flow velocity maps from the motion of stream floaters. Such dronebased surface flow velocity estimates are compared to benchmark values from a current meter to demonstrate the validity of airborne flow velocimetry.

Our method is expected to help in hydrological monitoring in ungauged areas by providing information on the kinematics of surface waters. Differently from standard drone-based monitoring where image mosaicing techniques are used to assemble maps [30], our approach treats captured videos as quantitative data and analyzes them to measure the surface flow velocity. These observations could leverage current knowledge on the contribution of surface flows to the overall hydrological response of natural systems. Further, continuous technological advancements in battery life and camera storage capacity may foster observations at the catchment scale and in large scale and yet ungauged water systems, thus opening novel research avenues in hydrology.

The rest of the technical note is organized as follows. In Section 2, the aerial sensing platform, experimental settings, and image-based procedures are presented. In Section 3, we report experimental findings for the hovering assessment and the airborne flow velocimetry. In Section 4, we discuss the advancements of the proposed approach. Section 5 is left for conclusions.

\section{Materials and Methods}

Here, we present the aerial sensing platform utilized in the experiments. Further, we provide details for the hovering assessment and the airborne flow 
velocimetry experiments.

\subsection{Aerial Sensing Platform}

The aerial sensing platform features a DJI Phantom 2 quadrotor [31] mounting a Zenmuse H3-2D gimbal and a GoPro Hero 3 camera oriented with its axis along the perpendicular. This configuration allows for compensating the drone vibrations about the pitch and roll axes, while minimizing distortions in video capture due to the inclination of the camera axis with respect to the field of view (FOV). Remote photometric calibration is enabled through four green lasers $(532 \mathrm{~nm}$ in wavelength and less than $5 \mathrm{~mW}$ in power) installed at the four corners of the fuselage along the drone's yaw axis. The platform is less than $1.5 \mathrm{~kg}$ in weight and its overall cost is $€ 1,300$.

The relative distances of the laser pointers are measured upon assembly of the sensing platform with a precision caliper. This system of lasers has the twofold objective of: i) focusing points at known distances in the FOV for remote image calibration and ii) indicating possible elevation and attitude changes during hovering from the relative distance of the laser traces in the images [17]. With respect to i), the traces of the lasers in captured videos are used to estimate pixel dimensions in metric units. With respect to ii), inaccurate hovering leads to images that depict slightly different FOVs. However, high-speed cross-correlation should be applied on images displaying consistent regions of the fluid domain [32]. To this aim, lasers can be instrumental to automatically identify portions of footage captured while the drone is hovering at a given location. In fact, the traces that lasers determine on the ground vary, based on the elevation and attitude of the drone; therefore, sequences of images displaying similar traces can be directly processed 
for surface flow velocity estimation.

\subsection{Hovering Assessment}

A preliminary experiment assessment is conducted to assess the feasibility of using a commercial low-cost platform for airborne flow velocimetry. The aerial sensing platform presents limited hovering capability (vertical: $\pm 0.8 \mathrm{~m}$ and horizontal: $\pm 2.5 \mathrm{~m}[31])$. Therefore, airframe changes in attitude and elevation may lead to variable image FOVs $[33,34]$. To evaluate such FOV variations, we fly the platform in the hovering mode above a large scale grid in an outdoor facility at the University of Tuscia, Italy. We perform a $22 \mathrm{~s}$ flight, whereby the onboard GoPro frame rate is set to $60 \mathrm{~Hz}$ and the FOV to medium (focal length equal to $21 \mathrm{~mm}$ ). The experiment is conducted at dusk and during light air wind conditions $(2.19 \mathrm{~km} / \mathrm{h}$ wind speed) [35].

Hovering capability is assessed by estimating the relative variations in the pixel area of the grid cells captured by the drone during the flight. Slight variations in the pixel areas of the grid cells during the flight would suggest that changes in the drone's elevation and attitude can be mitigated with minimal image processing, and, therefore, drone-based videos are compatible with accurate surface flow observations. To provide a reliable assessment, the experimental time is set to $22 \mathrm{~s}$, whereas airborne flow velocimetry tests only last up to few seconds ( given the high camera frame rate, video data recorded in few seconds are largely sufficient for accurate measurements).

\subsubsection{Hovering Assessment Outdoor Facility}

The drone is flown above a $5 \times 5 \mathrm{~m}^{2}$ flat grid assembled in the outdoor laboratory at University of Tuscia, Italy, out of a dark opaque ground cloth 
and a structure of white strings. The strings are laid to create $1 \times 1 \mathrm{~m}^{2}$ squared grid cells. The dark cloth minimizes reflections and enhances the visibility of the white strings against the background. Figure 1 presents a schematics of the setup. The drone is deployed to hover above the center of the grid. The dashed red rectangle in Figure 1 shows the FOV captured by the onboard camera. The laser-focused rectangle is indicated in yellow.

\subsubsection{Image-based Hovering Evaluation}

Captured video data are preliminary corrected for the camera lens distortion. The video fish eye distortion is removed through the freely available GoPro Studio 2.0 Software. Then, the video is converted and decompressed to extract Full HD frames. Image processing entails a preliminary phase in which frames are converted to grayscale based on luminance information and then converted to binary by imposing a threshold. Image segmentation is then applied on binary images to retain essential features.

To estimate the pixel area of the grid cells, an artificial binary template depicting a representative node of the grid is created. Normalized crosscorrelation is performed between the template and images extracted from the experimental video. By retaining locations presenting high values of the cross-correlation coefficient and repairing false correlations, the pixel locations of the grid nodes are obtained. Image-based algorithms [36, 37] are utilized to compute the areas of the grid cells and the area of the rectangle identified by the four lasers. 


\subsection{Airborne Flow Velocimetry}

To ultimately demonstrate the potential of drone-based flow observations, surface flow measurements are conducted over a less than $1 \mathrm{~m}$-wide and $11 \mathrm{~cm}$-deep mountainous stream in the Rio Cordon natural catchment, Italy [18].

\subsubsection{Airborne Flow Velocimetry Study Site}

The Rio Cordon drains a $7.68 \mathrm{~km}^{2}$ natural basin located in the Dolomites, Northeastern Italy. The stream is a tributary of the Fiorentina stream that in turn flows into the Rio Cordevole. The catchment drainage network extends for approximately $19 \mathrm{~km}$ at an average slope of $47.85 \%$. Tests are executed at a local gauging station, which is equipped with water gauges, a coarse sediment grille, and a diversion pool for water and finer material. The gauging station is located at $1763 \mathrm{~m}$ above sea level where the area drained by the stream is approximately $5 \mathrm{~km}^{2}$ large. At the time of the experiments, a nearby meteorological station recorded a wind speed of $0.9 \mathrm{~m} / \mathrm{s}$.

\subsubsection{Airborne Flow Velocimetry Procedure}

The drone is flown above an artificially channeled stream reach located in the proximity of a stream gauge. The selected stream reach is a rectilinear tract with a concrete rectangular section that extends for approximately $10 \mathrm{~m}$. Surface velocity is measured using both artificial and natural tracers. Ten repetitions are performed for each type of tracer. Similar to [38, 39], artificial tracers are high-visibility in-house fabricated particles synthesized from biocompatible and buoyant children-friendly dough. Particle size ranges from 0.5 up to $1 \mathrm{~cm}$, and beads are red, yellow, orange, and green in color. 
Also, experiments are conducted using natural leaves as tracers. In both classes of experiments, the drone captures Full $\mathrm{HD}$ videos at $60 \mathrm{~Hz}$ frame rate. On average, the FOV extends for $9 \times 5 \mathrm{~m}^{2}$.

Drone-based data are compared to benchmark flow velocities obtained using an OTT C2 small current meter. Current meters yield accurate and repeatable open channel flow measurements up to a few centimeters from the water surface and, differently from more costly non-contact radars, are widely used by researchers and practitioners in environmental monitoring. In the experiments, the instrument is set to the time measurement mode, whereby the number of impulses recorded in $10 \mathrm{~s}$ are counted and related to flow velocity. The velocity is measured at a cross-section of the stream a few centimeters upstream the subarea used for comparing the drone tests. Benchmark values are obtained by measuring the flow at $0.5 \mathrm{~m}$ from the right stream bank (that is, in the center of the stream) and $3 \mathrm{~cm}$ underneath the water surface and averaging over three repetitions. Obtained values are relative to the uppermost layer of the stream (top 30\% of the stream depth), where the velocity is less influenced by wind effects and, therefore, it is likely higher than surface velocity.

\subsubsection{Airborne Flow Velocimetry Video Processing}

Similar to Section 2.2.2, drone-based videos are fish-eye undistorted and sequences of Full HD images are extracted. Similar to the image-based hovering evaluation, lasers' traces onto the stream surface are utilized to manually identify stable image sequences within videos captured from the drone. The lasers afford remote photometric calibration of experimental images. Specifically, the pixel distance between lasers traces is manually estimated in several 
frames and validated with respect to objects of known dimensions that are visible in the field of view.

Untrimmed images depicting the transit of the tracers are processed using the high-speed cross-correlation edPIV software [40], where frame acquisition rate is set to $60 \mathrm{~Hz}$, interrogation window size to $32 \times 32$ pixels, and grid size to $16 \times 16$ pixels. On average, the pixel area displaying the surveyed stream reach corresponds to $20 \%$ of the picture. Surface flow velocity maps are developed by averaging velocity estimations in time over the sequence of images analyzed in the experiment.

Due to imperfect hovering of the drone during image acquisition, image portions lying outside the stream presented non-null velocities. To remove such false readings, the average velocity of nodes outside the stream is automatically computed and subtracted from the entire set of velocity values. Given the stability of the aerial platform and the short duration of experimental flights, airframe vibrations do not excessively impact velocity estimation. In particular, velocities estimated without subtracting values of nodes outside the flow are 5-8\% larger than measurements herein reported. Comparison between measurements executed with the two classes of tracers is performed by considering a subarea of the stream that is consistently captured in each video. Then, time-averaged velocities are computed over the frame sequence and the maximum value of the velocity field in the subarea is used for comparison. 


\section{Results}

\subsection{Hovering Assessment}

Analysis of images recorded during the hovering test demonstrates that the platform stability is compatible with airborne flow velocimetry. During the flight time, consistent portions of the grid are captured by the camera. Specifically, the 11 grid cells enclosing colored markers in the left panel of Figure 2 are always visible in frames during the flight. Colored markers inside such grid cells indicate the average pixel area of each cell over the flight time. Squares 2, 5, 6, 9, and 10 present smaller areas than squares on the left hand side of the grid, suggesting that the drone is closer to the left portion of the grid.

In Figure 2 (right), the time series of the areas of the grid cells (colored dots) and of the laser focused rectangle (black dots) are shown. Due to illumination conditions, the trace of the four laser beams on the grid is not always visible ( $76 \%$ of the total number of frames depicted four laser traces). Notably, the cell areas slightly vary in time, with a maximum coefficient of variation of $0.89 \%$. On the other hand, external vibrations lead to a coefficient of variation as large as $2.94 \%$ in the time series of the area identified by the lasers. This is due to the fact that lasers are directly installed on the drone fuselage, whereas the camera gimbal efficiently mitigates the drone's vibrations, thus abating the coefficient of variation to less than a third of its value. We remark that improved stability is crucial for correlation-based flow velocity estimation, where the similarities between consecutive images are used to infer the displacement of depicted objects. Therefore, slight changes in the image FOV due to the drone's vibrations while hovering suggest that 
reliable cross-correlation is feasible.

\subsection{Airborne Flow Velocimetry}

Proof of concept velocity observations in the Rio Cordon show the potential of the drone-based approach for surface flow measurement. Aerial platform velocities measured in the subarea located in between the dark crosssectional stripe and the dark inclined stripes (approximately $0.76 \times 0.83 \mathrm{~m}^{2}$ ), Figure 3 (left), are in line with benchmark values, Table 1. Underestimations with respect to the impeller flowmeter readings may be due to the fact that the flowmeter is slightly below the water surface $(3 \mathrm{~cm}$ out of the total depth of $11 \mathrm{~cm}$ ), where wind effects are negligible and velocities are expected to be larger than surface flows.

With regards to the two sets of drone measurements in Table 1, differences between them are not statistically different as determined through one-way ANOVA $(F=2.43$ and $p=0.14)$. The slightly higher uncertainty obtained in case of natural floaters is attributed to the fact that they were heavily deployed in the stream and, therefore, involved longer flights. Therefore, since the hovering performance of the drone is reduced over longer flight times, the use of natural tracers yields the higher standard deviation in Table 1.

As shown in the velocity map in Figure 3 (left), the aerial platform is successful in monitoring extended portions of the stream and the flow physics across the stream is accurately captured, Figure 3 (right). Maps from the drone are slightly affected by local water reflections and areas of poor illumination, since the onboard camera offers extended FOVs with diffused, rather than direct, illumination. 


\section{Discussion and Remarks}

Here, we propose a novel surface flow observation concept based on a stand-alone sensing platform that integrates drone technology and optical methods. Not only are image data captured from the platform, but a system of lasers enables remote photometric calibration, thus circumventing the need for time-consuming and expensive field campaigns for GRPs acquisition. Specifically, in this study, a laser system is utilized for pixel calibration and for flow velocimetry analyses. Indeed, the stationarity of the time series of the area identified by the lasers is used to isolate stable sequences for flow measurements, whereby small variations are associated with instances of stable hovering.

Notably, the laser apparatus paves the way for unsupervised rapid observations in large scale hydrological systems. Indeed, instances of airborne flow velocimetry currently rely on the acquisition of fixed objects in the FOV for photometric calibration and for video stabilization due to imperfect hovering $[41,42,43]$. On the other hand, the use of the laser system will leverage pixel calibration in the absence of fixed reference objects, thus enabling low cost observations in large scale systems, such as estuaries and limnological environments. In such large scale settings that may exceed the maximum distance range allowed by the remote control, a priori planned GPS waypoint navigation may facilitate observations.

Beyond pixel calibration and video stabilization, analysis of the laser's traces in the FOV can be used to complement onboard built-in altimetry measurements. For instance, a smaller area corresponds to a higher distance of the drone from the ground and, thus, to the drone increasing its vertical 
position. Therefore, changes in the area and shape of the laser's traces on the ground can help in estimating the altitude of the drone with respect to the ground, thus opening the way for refined photometric calibration and enhanced measurement accuracy.

The presented system is inherently suited for real-time analysis in ungauged environments. Data acquisition is feasible in rather limited intervals of time (few seconds) and surface flow velocity map generation is an unsupervised process that can be afforded in 5-10 minutes. In addition, given the limited cost of the equipment and its ease of implementation (medium flight skills can be achieved after some hours of training), the approach is versatile and likely sustainable for most research groups. With regards to the use of tracers, even if highly-visible artificial tracers enable rapid velocity estimations, their use requires either the presence of operators or installation of remotely-operated devices to deploy the particles [18].

The dependence of LSPIV measurements on the presence of homogeneously distributed tracers may pose a significant technical challenge to practical implementations of the proposed approach. To overcome this issue, we envision the development of machine learning techniques to automatically de-noise pictures [44] and to emphasize floaters' contrast against the image background. Alternative image-based flow velocimetry algorithms that yield the trajectory of individual floaters, such as particle tracking velocimetry, should be explored [45]. In the case of floods or severe events, naturallyoccurring debris and sediments may be sufficient to obtain surface flow velocity measurements [46].

The proposed approach may be readily implemented to provide fully 
remote and rapid flow discharge estimates in inaccessible areas and large scale ecosystems. For instance, in [47, 48, 49], an entropy-based method is proposed to estimate flow discharge based on the maximum velocity observed on the stream surface. This approach establishes a linear relationship between the maximum surface velocity and mean velocity in natural rivers $[50,51]$. The integration of such entropy-based approach and droneenabled distributed surface velocity observations may lead to flow discharge estimates in complex environments. Further, application of image mosaicking and FOV reconstruction techniques may enable surface flow velocity gauging over large water areas.

\section{Conclusions}

In this work, we demonstrated the potential of integrating an aerial platform and optical sensing for surface flow measurements in natural environments. We designed the aerial platform to enable completely remote measurements, thus circumventing the need for on site surveys that are typically required by traditional measurement techniques, and enabling novel observations in inaccessible areas. By investigating the hovering capability of the aerial platform during a flight of more than $20 \mathrm{~s}$, we found that the approach is suitable for remote flow observations. Further, surface flow velocity measurements performed from the drone on a small scale mountainous stream were in good agreement with data from on site flow sensing systems.

We expect drones to be a forward-looking addition to the experimental toolbox currently available to hydrologists and geophysicists. As technology progresses toward better performing drones, current limitations, such 
as imperfect hovering capabilities, constrained flight time and payload, will be easily overcome, and this approach should enable new avenues in hydrological observations. Future studies will be devoted to the application of the presented methodology to the kinematic characterization of alternative environmental settings, such as channel and rill flows.

\section{Acknowledgements}

This work was supported by the Ministero degli Affari Esteri project 2015 Italy-USA PGR00175, by the American Geophysical Union Horton (Hydrology) Research Grant for Ph.D. students, by the UNESCO Chair in "Water Resources Management and Culture", and by the National Science Foundation under grant numbers BCS-1124795 and CMMI-1129820.

[1] G. E. Tucker, R. L. Bras, Hillslope processes, drainage density, and landscape morphology, Water Resources Research 34 (10) (1998) 27512764 .

[2] A. J. Boulton, S. Findlay, P. Marmonier, E. H. Stanley, H. M. Valett, The functional significance of the hyporheic zone in streams and rivers, Annual Review of Ecology and Systematics 29 (1998) 59-81.

[3] V. T. Chow, D. R. Maidment, L. W. Mays, Applied Hydrology, McGrawHill, 1988.

[4] L. Mari, E. Bertuzzo, L. Righetto, R. Casagrandi, M. Gatto, I. Rodriguez-Iturbe, A. Rinaldo, Modelling cholera epidemics: the role of waterway, human mobility and sanitation, Journal of the Royal Society Interface 9 (103) (2012) 376-388. 
[5] M. Hrachowitz, H. H. G. Savenije, G. Blöschl, J. J. McDonnell, M. Sivapalan, J. W. Pomeroy, B. Arheimer, T. Blume, M. P. Clark, U. Ehret, F. Fenicia, J. E. Freer, A. Gelfan, H. V. Gupta, D. A. Hughes, R. W. Hut, A. Montanari, S. Pande, D. Tetzlaff, P. A. Troch, S. Uhlenbrook, T. Wagener, H. C. Winsemius, R. A. Woods, E. Zehe, C. Cudennec, A decade of predictions in ungauged basins (PUB) a review, Hydrological Sciences Journal 58 (6) (2013) 1198-1255.

[6] A. Tazioli, Experimental methods for river discharge measurements: comparison among tracers and current meter, Hydrological Sciences Journal 56 (7) (2011) 1314-1324.

[7] O. Planchon, N. Silvera, R. Gimenez, D. Favis-Mortlock, J. Wainwright, Y. Le Bissonnais, G. Govers, An automated salt-tracing gauge for flowvelocity measurement, Earth Surface Processes and Landforms 30 (7) (2005) 833-844.

[8] T. H. Yorke, K. A. Oberg, Measuring river velocity and discharge with acoustic Doppler profilers, Flow Measurement and Instrumentation 13 (5-6) (2002) 191-195.

[9] H. Chanson, Hydraulics of Open Channel Flow, Elsevier ButterworthHeinemann, Oxford,UK, 2004.

[10] C. Leibundgut, P. Maloszewski, C. Külls, Tracers in Hydrology, WileyBlackwell, Oxford, 2009.

[11] J. Fulton, J. Ostrowski, Measuring real-time streamflow using emerg- 
ing technologies: radar, hydroacoustics, and the probability concept, Journal of Hydrology 357 (1-2) (2008) 1-10.

[12] W. J. Plant, W. C. Keller, K. Hayes, Measurement of river surface currents with coherent microwave systems, Geoscience and Remote Sensing, IEEE Transactions on 43 (6) (2005) 1242-1257.

[13] A. Tarpanelli, S. Barbetta, L. Brocca, T. Moramarco, River discharge estimation by using altimetry data and simplified flood routing modeling, Remote Sensing 5 (9) (2013) 4145-4162.

[14] I. Fujita, M. Muste, A. Kruger, Large-scale particle image velocimetry for flow analysis in hydraulic engineering applications, Journal of Hydraulic Research 36 (3) (1997) 397-414.

[15] M. Muste, I. Fujita, A. Hauet, Large-scale particle image velocimetry for measurements in riverine environments, Water Resources Research 44 (4) (2008) W00D19.

[16] A. Hauet, A. Kruger, W. Krajewski, A. Bradley, M. Muste, J. Creutin, M. Wilson, Experimental system for real-time discharge estimation using an image-based method, Journal of Hydrologic Engineering 13 (2) (2008) $105-110$.

[17] F. Tauro, M. Porfiri, S. Grimaldi, Orienting the camera and firing lasers to enhance large scale particle image velocimetry for streamflow monitoring, Water Resources Research 50 (9) (2014) 7470-7483.

[18] F. Tauro, S. Grimaldi, A. Petroselli, M. Porfiri, Fluorescent particle 
tracers in surface hydrology: a proof of concept in a natural stream, Water Resources Research 48 (6) (2012) W06528.

[19] F. Tauro, S. Grimaldi, A. Petroselli, M. C. Rulli, M. Porfiri, Fluorescent particle tracers in surface hydrology: a proof of concept in a semi-natural hillslope, Hydrology and Earth System Sciences 16 (8) (2012) 2973-2983.

[20] E. Palermo, Drones could grow to $\$ 11$ billion industry by 2024 , livescience.com.

[21] T. Clarke, Pilotless research aircraft: Flying free, Nature 417 (2002) $582-583$.

[22] J. Cohen, Drone spy plane helps fight California fires, Science 318 (5851) (2007) 727 .

[23] A. Eltner, P. Baumgart, H.-G. Maas, D. Faust, Multi-temporal UAV data for automatic measurement of rill and interrill erosion on loess soil, Earth Surface Processes and Landforms 40 (6) (2015) 741-755.

[24] A. Pérez-Alberti, A. S. Trenhaile, An initial evaluation of drone-based monitoring of boulder beaches in Galicia, north-western Spain, Earth Surface Processes and Landforms 40 (1) (2014) 105-111.

[25] A. J. S. McGonigle, A. Aiuppa, G. Giudice, G. Tamburello, A. J. Hodson, S. Guerrieri, Unmanned aerial vehicle measurements of volcanic carbon dioxide fluxes, Geophysical Research Letters 35 (6) (2008) L06303.

[26] L. Shelley, L. Knuth, J. J. Cassano, Estimating sensible and latent heat fluxes using the integral method from in situ aircraft measurements, 
Journal of Atmospheric and Oceanic Technology 31 (9) (2014) 19641981.

[27] R. Schiffman, Drones flying high as new tool for field biologists, Science 344 (6183) (2014) 459.

[28] J. Jensen, Biophysical remote sensing, Annals of the Association of American Geographers 73 (1) (1983) 111-132.

[29] J. S. Famiglietti, A. Cazenave, A. Eicker, J. T. Reager, M. Rodell, I. Velicogna, Satellites provide the big picture, Science 349 (6249) (2015) 684-685.

[30] W. W. Immerzeel, P. D. A. Kraaijenbrink, J. M. Shea, A. B. Shrestha, F. Pellicciotti, M. F. P. Bierkens, S. M. deJong, High-resolution monitoring of Himalayan glacier dynamics using unmanned aerial vehicles, Remote Sensing of Environment 150 (2014) 93-103.

[31] http://www.dji.com/ (2014).

[32] M. Raffel, C. E. Willert, S. T. Wereley, J. Kompenhans, Particle Image Velocimetry. A practical guide., Springer, New York, 2007.

[33] I. Fujita, T. Hino, Unseeded and seeded PIV measurements of river flows video from a helicopter, Journal of Visualization 6 (3) (2003) 245-252.

[34] I. Fujita, Y. Kunita, Application of aerial LSPIV to the 2002 flood of the Yodo River using a helicopter mounted high density video camera, Journal of Hydro-Environment Research 5 (4) (2011) 323 - 331. 
[35] http://www.wmo.int (2015).

[36] J. L. Coolidge, A historically interesting formula for the area of a quadrilateral, American Mathematical Monthly 46 (1939) 345-347.

[37] R. S. Hunter, Photoelectric color difference meter, Journal of the Optical Society of America 48 (12) (1958) 985-993.

[38] F. Tauro, M. Porfiri, S. Grimaldi, Fluorescent eco-particles for surface flow physics analysis, AIP Advances 3 (3) (2013) 032108.

[39] F. Tauro, E. Rapiti, J. F. Al-Sharab, L. Ubertini, S. Grimaldi, M. Porfiri, Characterization of eco-friendly fluorescent nanoparticle doped-tracers for environmental sensing, Journal of Nanoparticle Research 15 (9) (2013) 1884.

[40] L. Gui, EDPIV - Evaluation Software for Digital Particle Image Velocimetry, http://legui.net (2013).

[41] M. Detert, V. Weitbrecht, A vehicle airborne velocimetry system: proof of concept, Journal of Hydraulic Research 53 (4) (2015) 532.

[42] F. Tauro, C. Pagano, P. Phamduy, S. Grimaldi, M. Porfiri, Largescale particle image velocimetry from an unmanned aerial vehicle, IEEE/ASME Transactions on Mechatronics 20 (6) (2015) 3269-3275.

[43] F. Tauro, A. Petroselli, E. Arcangeletti, Assessment of drone-based surface flow observations, Hydrological Processes 30 (7) (2015) 1114-1130.

[44] F. Tauro, S. Grimaldi, M. Porfiri, Unraveling flow patterns through nonlinear manifold learning, PLoS ONE 9 (3) (2014) e91131. 
[45] F. Tauro, A. Petroselli, M. Porfiri, L. Giandomenico, G. Bernardi, F. Mele, D. Spina, S. Grimaldi, A novel permanent gauge-cam station for surface flow observations on the Tiber river, Geoscientific Instrumentation, Methods and Data Systems Under review.

[46] F. Tauro, G. Olivieri, A. Petroselli, M. Porfiri, S. Grimaldi, Flow monitoring with a camera: a case study on a flood event in the Tiber River, Environmental Monitoring and Assessment 188 (2) (2015) 118.

[47] C.-L. Chiu, Entropy and probability concepts in hydraulics, Journal of Hydraulic Engineering 113 (5) (1987) 583-599.

[48] C.-L. Chiu, Entropy and 2-D velocity distribution in open channels, Journal of Hydraulic Engineering 114 (7) (1988) 738-756.

[49] G. Farina, S. Alvisi, M. Franchini, T. Moramarco, Three methods for estimating the entropy parameter $\mathrm{M}$ based on a decreasing number of velocity measurements in a river cross-section, Entropy 16 (5) (2014) $2512-2529$.

[50] C.-L. Chiu, Application of entropy concept in open channel flow study, Journal of Hydraulic Engineering 117 (5) (1991) 615-628.

[51] R. Xia, Relation between mean and maximum velocities in a natural river, Journal of Hydraulic Engineering 123 (8) (1997) 720-723. 


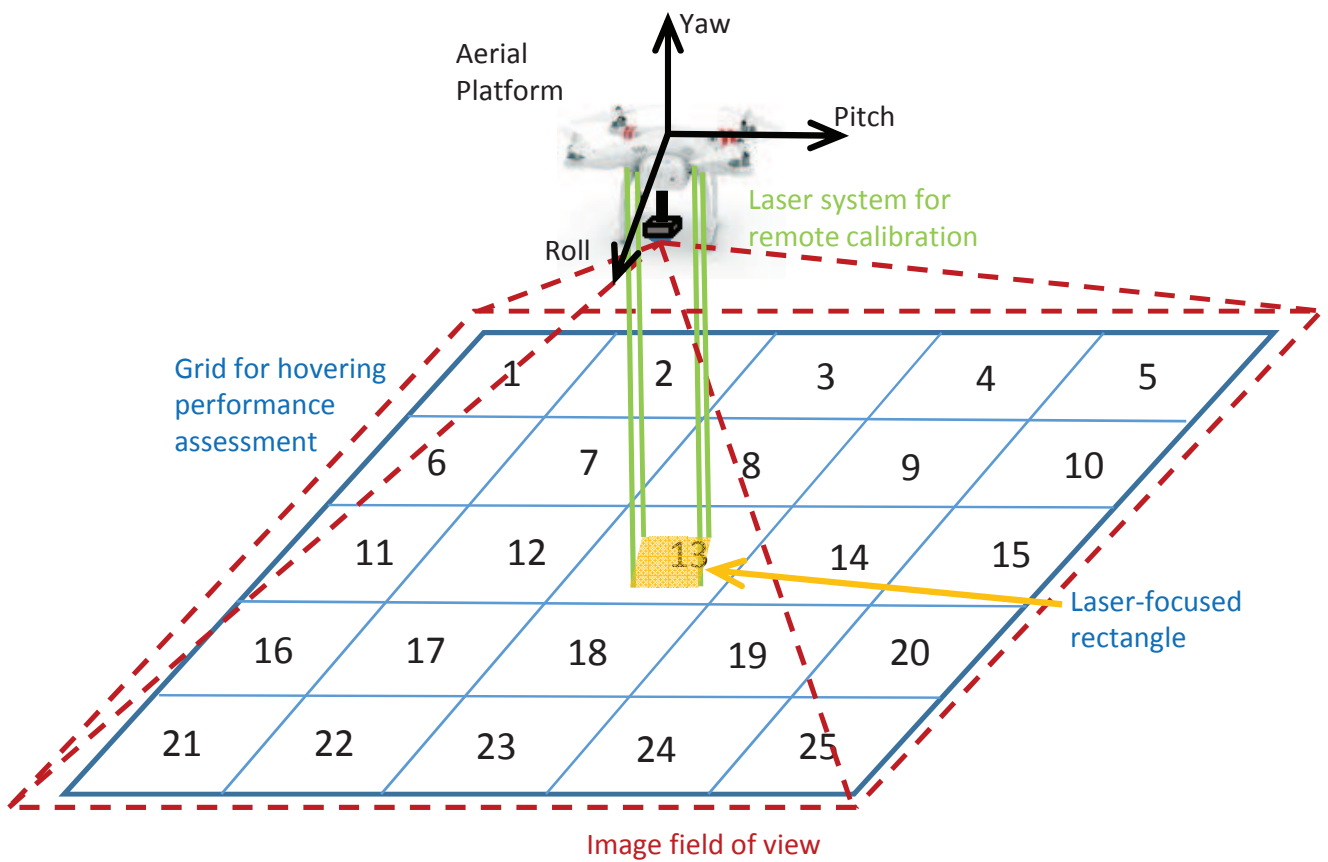

Figure 1: Sketch of the experimental setup. Analysis of the squares of the grid was used to assess the ability to capture stable videos.

Table 1: Velocity measurements performed on the natural stream. Symbols $v_{\mathrm{m}}$ and $\sigma$ stand for average maximum velocity and standard deviation, respectively. Average maximum velocities and standard deviations from the drone are obtained from LSPIV time-averaged maps by averaging over the yellow dashed subarea reported in Figure 3.

$$
v_{\mathrm{m}}[\mathrm{m} / \mathrm{s}] \quad \sigma[\mathrm{m} / \mathrm{s}]
$$

\begin{tabular}{lll}
\hline Flowmeter & 2.54 & 0.09 \\
\hline
\end{tabular}

Drone

$\begin{array}{lll}\text { artificial tracers } & 2.29 & 0.09 \\ \text { natural tracers } & 2.15 & 0.27\end{array}$



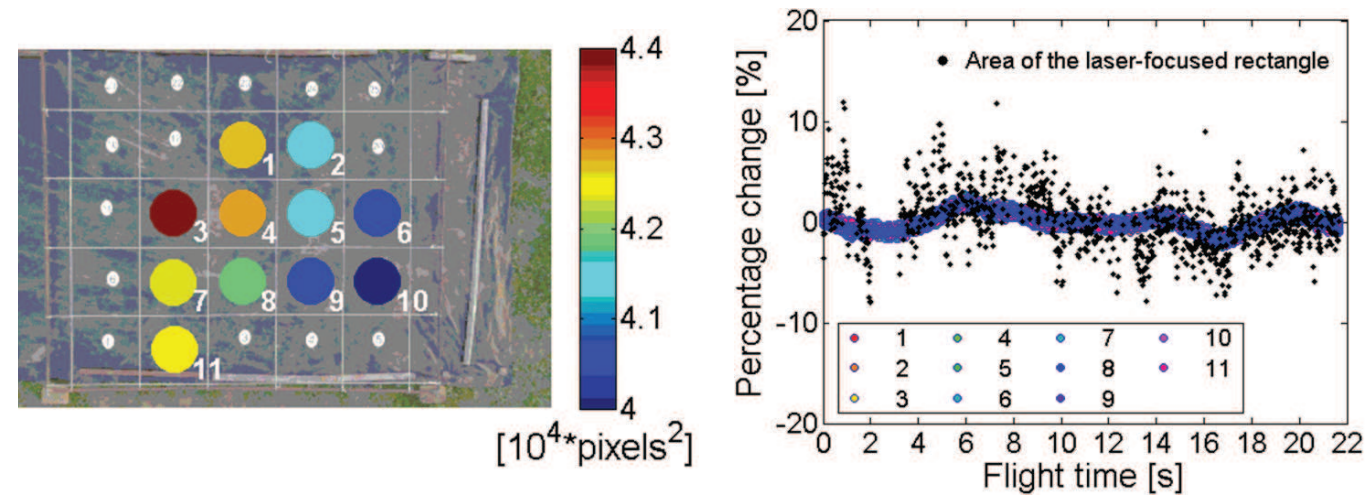

Figure 2: Left, experimental grid for assessing the drone's hovering capability. Colored markers inside each square indicate the average area in pixels. Right, time histories of the area of each of the 11 squares visible during the flight (colored dots), and of the area of the rectangle focused by the laser beams (black dots). Raw data are smoothed through a local regression filter with weighted linear least squares and a parabolic model. 

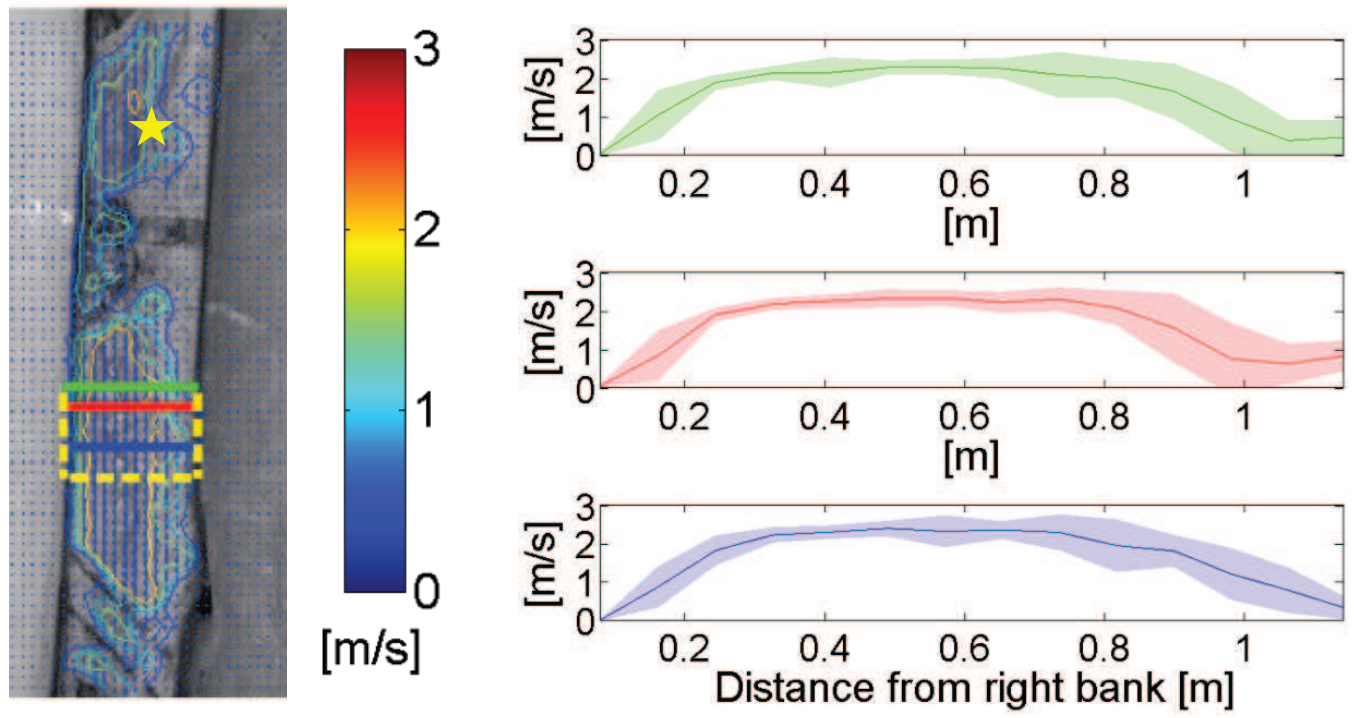

Figure 3: Left, representative surface flow velocity maps from the aerial platform based on the use of natural floaters. The yellow star indicates the location at which measurements with the impeller flowmeter are taken. Right, time-averaged cross-sectional profiles corresponding to the green, red, and blue cross-sections highlighted in the map. Shaded areas indicate standard deviations. The yellow dashed box in the left panel highlights the subarea used for measurement comparison. 